\title{
Eine kleine Eschatologie?
}

\author{
Betrachtungen zum Roman Aller Tage Abend von Jenny \\ Erpenbeck
}

Monika Hirt

"Wer sind wir? Wo kommen wir her? Wohin gehen wir? Was erwarten wir? Was erwartet uns? " Das sind die Fragen, mit denen Ernst Bloch sein Prinzip Hoffnung eröffnet. ${ }^{1}$ Und es sind genau diese Fragen, denen auch Jenny Erpenbeck in ihrem 2012 erschienenen Roman Aller Tage Abend ${ }^{2}$ nachgeht. Fragen, an denen sich ihre Romanfiguren reiben - nicht abstrakt, sondern äusserst lebensnah; pragmatisch, und dennoch mit ausserordentlichem Tiefgang.

Der Roman besteht aus fünf Büchern, welche jeweils einen Lebensabschnitt der Protagonistin von der Geburt im Jahre 1902 bis zu ihrem Tod als 90-Jährige erzählen. Das Besondere daran ist, dass die Hauptfigur nach jedem Abschnitt stirbt: als Säugling in einer jüdischen Familie in Galizien am plötzlichen Kindstod; durch Freitod als junge, von der Liebe enttäuschte Frau in Wien kurz nach dem Ersten Weltkrieg; als Kommunistin im sibirischen Straflager; als gefeierte DDR-Schriftstellerin durch einen Unfall und schliesslich, nach der Wende, als zunehmend demente 90-Jährige im Altersheim. Den ersten vier Büchern folgt jeweils ein Zwischenkapitel, ein sogenanntes Intermezzo. In diesem wird die Geschichte mit einem "Wäre jedoch" oder "Hätte sie jedoch" jeweils so variiert, dass die Protagonistin doch nicht zu Tode kommt, sondern weiterlebt. Erst am Ende des fünften Buches stirbt sie endgültig.

\section{Ohne Tod lässt sich das Leben nicht verstehen}

Mit diesen vielen Leben, die hier in einem Leben enthalten sind, bringt Jenny Erpenbeck die Lesenden mit all den entscheidenden Fragen des Lebens in Berührung. Aber warum lässt die Autorin die Protagonistin dafür immer wieder sterben? Sie tut es, weil der Tod elementar ist. Weil die Fragen rund um die eigene Existenz und Identität erst im Hinblick auf den Tod - als Begrenzung irdischen

1 Ernst Bloch, Das Prinzip Hoffnung, Berlin 1959, 1.

2 Jenny Erpenbeck, Aller Tage Abend, München ${ }^{2} 2012$. 
Lebens - bedeutungsvoll werden. Gemäss Eberhard Jüngel kann sich der Lebende ohne den Tod nicht verstehen. Und umgekehrt lässt sich der Tod nur über das Leben begreifen. ${ }^{3}$ Genau das demonstriert Jenny Erpenbeck anhand der verschiedenen Lebensstationen und wechselnden Sterbesituationen.

Dass die Antworten kaum etwas anderes sein können "als wiederum - Fragen ${ }^{4}$, dessen ist sich Jenny Erpenbeck nur zu bewusst. ${ }^{5}$ Als Schriftstellerin nutzt sie die Freiheit, diese Fragen in ihrer ganzen Komplexität und Vielschichtigkeit auszuloten und mehrere Perspektiven einzunehmen: Fünf unterschiedliche Tode in fünf verschiedenen Lebensphasen und Epochen ergeben ein facettenreiches Panoptikum von Todesarten sowie Lebens- und Jenseitsvorstellungen. Aber das ist nur der eine Aspekt dieses Erzählkonzepts. Mit ihrer narrativen Pluralisierung zielt sie tiefer - direkt ins Zentrum existenzieller Fragen hinein: Warum entwickelt sich ein Leben so und nicht anders? Warum sterben die einen früh und die anderen spät? Was wäre gewesen, wenn ...? Jenny Erpenbeck nimmt diese Fragen, die im Normalfall als müssig abgetan werden, beim Wort und greift in das unveränderbare Schicksal ein: Das Leben hätte auch anders verlaufen können und tut es hier auch. Die Autorin lässt die ungelebten Möglichkeiten, das, was hätte werden können, zur Realität werden. ${ }^{6}$ Damit ignoriert sie die $"$ Diskrepanz zwischen der Wirklichkeit eines gelebten Lebens und den Möglichkeiten eines zu lebenden Lebens «? Sie setzt sich über die Grenze des Todes hinweg. Zumindest so lang, bis die Diskrepanz zwischen einem frühen Tod und einem natürlichen Alterstod aufgehoben ist.

Aber was ist damit gewonnen? Kommt die Protagonistin damit der Erfüllung ihrer Lebensträume näher? Ja und nein. Ja, weil sie mit jedem Lebensabschnitt um ein Stück Lebensgeschichte und Lebenserfahrung reicher wird. Nein, weil die meisten existenziellen

\footnotetext{
3 Eberhard Jüngel, Tod, Stuttgart ${ }^{3} 1973,23,25$.

4 Ebd., 25.

5 In einem Interview sagt Jenny Erpenbeck: „Ich bin froh, wenn es mir gelingt, die richtigen Fragen zu formulieren, überhaupt zu wissen, wo ich mit dem Fragen anfangen muss. Ich beantworte aber die Fragen weder selber, noch erwarte ich Antworten von den Lesenden."Neue Osnabrücker Zeitung (NOZ), 11.1.2013; http://www.noz.de/ deutschland-welt/medien/artikel/1307/1201-med-erpenbeck\#gallery \&0\&0\&1307 (18.04.2016).

6 In diesem Sinn lautet der erste Satz des Romans (11): „Der Herr hat's gegeben, der Herr hat's genommen, hatte die Grossmutter am Rande der Grube zu ihr gesagt. Aber das stimmte nicht, denn der Herr hatte viel mehr genommen, als da war - auch alles, was aus dem Kind hätte werden können, lag jetzt da unten und sollte unter die Erde." 7 Jüngel, Tod, 37 .
} 
Fragen rund um die Lebensverwirklichung bestehen bleiben. Der herausgezögerte Tod bedeutet nicht, dass die Begrenztheit menschlichen Lebens aufgehoben ist. In jedem Lebensabschnitt müssen sich die Protagonistin und ihre Angehörigen mit dieser Beschränkung, mit dem Tod und seinen Folgen auseinandersetzen. In vielen der geschilderten Lebenssituationen werden die Grenzen menschlicher Einflussnahme schmerzlich bewusst, und Illusionen werden zerstört. Mit dieser Erfahrung wächst die Erkenntnis, dass Erfüllung und Vollendung nicht in der Hand der Menschen liegen.

\section{Variationen über den Tod}

Jenny Erpenbeck breitet in ihrem Roman einen äusserst komplex geknüpften Erzählteppich aus, bei dem es einem als Interpretin schwer fällt, einem einzigen roten Faden zu folgen und sich auf bestimmte Erzählelemente zu beschränken. Jede Szene löst eine Kette von Assoziationen aus - sowohl intertextuelle Bezüge als auch eigene Gedanken und Bilder. Allein wie sie die fün Sterbeszenen beschreibt, ist meisterhaft. Nicht nur wegen der Kraft ihrer poetischen Sprache und der Fähigkeit, Emotionen und Atmosphäre zu vermitteln, sondern vor allem dadurch, dass ihre Darstellungen lebensechte Erfahrungen widerspiegeln. Sei es die Ohnmacht der jungen Mutter, nicht zu wissen, "was in solch einer Lage zu tun war" (12), als das Baby plötzlich nicht mehr atmete, weil "niemand ihr vorher gesagt hat, dass das Leben nicht funktioniert wie eine Maschine» (15); seien es die romantischen, idealisierenden Sterbephantasien der jungen 18-Jährigen, die beschliesst, "sich aus der Welt« zu nehmen, um endlich lieben zu dürfen, "wen immer sie lieben will, und sich dabei mitsamt ihren toten Gedanken ganz und gar in alle Anderen auflösen « zu können (122); seien es die berühmten letzten Sekunden, in denen sich das ganze Leben abspult, wie bei der Schriftstellerin, die sich, während sie die Treppe hinunterfällt, fragt: »Was ist das für ein Schlund, der sich da auftut und sämtliche Gedanken, die einer denken kann, in sich hineinsaugt, [...] und wo war dieser Schlund zuvor?" (213); seien es die Visionen der alten Frau, die im Vorfeld eines nahen Todes typisch sind: »Ich bin jetzt in einem Stadium des Übergangs" (257) sagt Frau Hoffmann zum Schattenriss, der in ihrem Zimmer sitzt und den sie als ihre Mutter wiedererkennt. Der toten Mutter, mit der sie Zeit ihres Lebens ein schwieriges Verhältnis hatte, kann sie ihre Ängste vor dem Sterben anvertrauen und wird getröstet: »Am Anfang der Woche, in der sie, einen Tag nach ihrem 
neunzigsten Geburtstag, sterben wird, lächelt Frau Hoffmann zum ersten Mal in ihrem Leben gemeinsam mit ihrer Mutter.« (258)

Genauso präsent wie der "eigene Tod « 8 ist in Aller Tage Abend der »Tod des anderen«. Denn der Tod der Protagonistin beeinflusst nachhaltig die Einstellungen der Angehörigen zu ihrem eigenen Leben, wie auch zum Leben und Sterben generell. Zusätzlich verschärft wird die individuelle Situation durch den "allgemeinen Tod», welcher vor allem in den ersten drei Büchern in Form von Verfolgung und Krieg immer präsent ist. Grundsätzlich wird bei allen fünf Lebensabschnitten deutlich, wie sehr die politische und gesellschaftliche Lage die individuelle Situation prägt und beeinflusst.

\section{Was bleibt?}

Wie mit dem Verlust umgehen? Die Angehörigen der verstorbenen Hauptfigur entwickeln unterschiedliche Strategien. Auffallend ist, dass die Männer öfter an der Situation verzweifeln und sogar die Flucht ergreifen, während die Frauen versuchen, möglichst schnell wieder ins Alltagsleben zurück zu finden. Denn, so die Erkenntnis der Mütter: »Ums Leben muss sich schliesslich auch jemand kümmern« (126), oder in Anlehnung an den Titel: "Am Ende eines Tages, an dem gestorben wurde, ist längst noch nicht aller Tage Abend.« (23) Dies gilt insbesondere in den Zeiten, in denen nicht nur das Leben, sondern das Überleben gesichert werden muss. Der Vater und die jüngere Schwester der jung Verstorbenen hingegen können nicht begreifen, dass sie die ganze Zeit arglos in den Tag hineingelebt haben, während die Ältere auf ihren Tod zusteuerte. Im Gegensatz zur Autorin können sie die Zeit jedoch nicht zurückschrauben und ihr eine andere Wendung gegeben, obwohl sie das am liebsten möchten:

"Bis zum Sonntag vor anderthalb Wochen war die Welt noch in Ordnung, sagt der Vater. Sicher, sagt die Mutter.

Irgendwann gegen Morgen ist sie am Montag doch, sagt der Vater, auch am Dienstag, sagt die jüngere Tochter [...], am Mittwoch habe ich, und dann, in der Nacht drauf, sagt die Kleine [...].

Und dann kam der Sonntag.

Hört doch auf, sagt jetzt die Mutter zu ihrem Mann und der Tochter, so macht ihr sie auch nicht wieder lebendig. [...]

\footnotetext{
8 Vgl. Die Typologie des Sterbens und des Todes, in: Klaus Feldmann, Tod und Gesellschaft. Sozialwissenschaftliche Thanatologie im Überblick, Wiesbaden 2010, 21.
} 
Was haben wir nur in Himmels Herrgotts Namen am Sonntagabend gemacht, fragt der Vater, und fängt an zu weinen.« (126)

Erst jetzt, wenn es zu spät ist, realisieren sie, wie wenig sie über die Tochter respektive Schwester gewusst haben. Von der Familie unverstanden, hatte diese sich ihrem Tagebuch anvertraut. Die Jüngere weiss, dass sich alles, was ihre Schwester ausgemacht hat und ihr wichtig war, in diesem Tagebuch findet. Diese einzige Verbindung, die ihr zu ihrer Schwester geblieben ist, will sie unbedingt retten, deswegen nimmt sie das Tagebuch mit auf die Flucht. Ihre Schwester so vor dem Vergessen zu bewahren, wurde ihr jedoch nicht vergönnt. Das zweite Buch endet traurig:

»Im Jahr 1944 wird in einem Birkenwäldchen ein Heft mit handschriftlichen Tagebuchaufzeichnungen auf die Erde fallen, als ein Wachposten eine junge Frau mit seinem Gewehrkolben vorwärts stösst, und sie sich mit den Armen, mit denen sie vorher das Heft an sich gedrückt hat, zu schützen versucht. Das Heft wird in den Dreck fallen, [...] das Heft wird ein Weilchen dort liegen, $[\ldots]$ bis alle Geheimnisse, die dort aufgeschrieben sind, die gleiche Farbe haben wie der Morast.« (132)

Was bleibt von einem Menschen über den Tod hinaus erhalten? Woran wird man sich erinnern? Diese Fragen sind für die Figuren zentral. Was aber als erinnerungswürdig angesehen wird, kann sehr unterschiedlich ausfallen. Das zeigt das vierte Buch, in dem die Protagonistin als gefeierte DDR-Schriftstellerin die Treppe hinunterstürzt und stirbt. Die Trauer des Sohnes um seine Mutter, seine Erinnerungen an sie, und die offiziellen staatlichen Nachrufe auf die verdiente Heldin des Sozialismus könnten unterschiedlicher nicht sein. Das illustrieren ineinander übergehende kontrastierende Abschnitte, wie dieser:

"Ist seine Mutter abgerutscht oder gestolpert? Auf welcher der Stufen lag denn ihr Kopf, als sie aufgehört hat zu atmen? Aber selbst wenn er alles über den letzten Moment wüsste, in dem seine Mutter gelebt hat, dann wüsste er dennoch nicht, was das hiess, dass sie jetzt tot war. Gestern wurde die grosse Künstlerin H., die Trägerin der Genosse-G.-Medaille, der Ehrennadel zum Grossen Vaterländischen Verdienstorden in Gold, des Goethe-Preises sowie zahlreicher anderer hoher und höchster Auszeichnungen unserer Republik, plötzlich und unerwartet aus dem Leben gerissen. Wir werden der tapferen Antifaschistin und der der Sache der Arbeiterklasse treu ergebenen Genossin H. auf ewig ein ehrendes Angedenken bewahren.« (217) 
Der Staat formuliert den Anspruch, das Vermächtnis der Genossin auf ewig zu erhalten. Ganz anders verhält es sich mit den letzten Gedanken der sterbenden Schriftstellerin und denjenigen ihres trauernden Sohnes. Beide beschäftigt, dass nun, durch den plötzlichen Tod, ganz viel verloren geht. Erinnerungen, die nichts mit dem öffentlichem Wirken zu tun haben, sondern Familiengeschichten und -traditionen beinhalten: Es sind kleine, scheinbar banale Dinge »Welche Apfelsorte ist für den Strudel am besten?» (222) -, die sich angesichts des Verlustes plötzlich als bedeutungsvoll entpuppen und für den Einzelnen einen grösseren Wert besitzen als gesellschaftliche Anerkennung. Die Gedanken des Sohnes machen deutlich: Das, woran sich der Staat auf ewig erinnern will, umfasst nur einen Bruchteil dessen, was die Verstorbene hinterlässt; genauso wie der menschliche Anspruch auf ewiges Andenken und Erinnern grundsätzlich eine Illusion ist. Der Tod macht schmerzlich bewusst:

"Mit ihrem Leben hören auch die Erinnerungen, die er an sie haben könnte, zu wachsen auf. [...] Aber durch seine Vergesslichkeit wird er die Mutter nun früher oder später noch ein zweites Mal verlieren, dann stückweis.» (220)

\section{Was wird sein?}

Der Kontrast von öffentlicher , Wahrheit und privater ist auch im dritten Buch zentral: Die Protagonistin lebt in Moskau und verfasst für ihren Antrag zur Aufenthaltsgenehmigung einen Lebenslauf. Ihr ist bewusst, dass es dabei nicht nur um eine simpler Aufenthalts-, sondern vielmehr um eine Daseinsberechtigung geht. Ihre Gedanken hüpfen zwischen parteikonformen Aussagen und Formulierungen, die die Empfänger wahrscheinlich über ihr Leben hören wollen und ihren ganz anders geprägten persönlichen Erinnerungen hin und her. Die Souveränität, selbst über ihr Leben zu entscheiden, hat sie in diesem totalitären System verloren:

"[E]s kann sein, dass dieser geschriebene Lebenslauf den Lauf ihres wirklichen Lebens beendet, dass dieses Schriftstïck, wenn man so will, sich in eine Waffe verwandelt, die sie sich selbst schreibt. Es kann auch sein, dass das Schriftstück aufbewahrt wird, und dass sie von dem Moment an, in dem sie es abgegeben hat, dagegen anleben muss [...].Vielleicht aber gelingt es ihr [...] und deshalb nur sucht sie so lange nach den richtigen Worten, vielleicht gelingt es ihr, sich mit dem Schreiben eine Rettung zu 
schreiben, und den Lauf ihres Lebens, durch ein paar Buchstaben mehr oder weniger, zu verlängern oder wenigstes zu erleichtern, auf nichts anderes kann sie hoffen, als darauf, sich durchs Schreiben ins Leben zurückzuschreiben. Aber was sind die richtigen Worte? Käme sie mit einer Wahrheit weiter als mit einer Lüge? Und welche der vielen möglichen Wahrheiten oder Lügen soll sie dann nehmen? Wenn sie doch nicht weiss, wer lesen wird, was sie schreibt.«(141f.)

Auch in diesem Lebensabschnitt weichen Lebensideale der Desillusionierung. Das Land, auf das sie ihre Hoffnungen gesetzt hat, von dem sie dachte, dass "der Tod hier keine Währung mehr« (185) ist, entpuppt sich als menschenverachtender, von Gewalt und Willkür zersetzter Staat.

Der Lebenslauf der Frau landet tatsächlich auf dem falschen Stapel; sie wird verhaftet und kommt in ein sibirisches Arbeitslager, wo sie an Entkräftung stirbt. Gleichheit und Gerechtigkeit - so will es die Ironie des Schicksals (oder vielmehr die Ironie der Autorin) erfährt sie erst im Jenseits:

»Und die Toten verbrennen in diesem Feuer [in dem im Straflager Schlacke verbrannt wird; $\mathrm{MH}]$ ) all die Sätze, die sie, als sie selbst noch Lebende waren, einmal gesagt haben - aus Angst, aus Überzeugung, aus Zorn, aus Gleichgültigkeit oder aus Liebe gesagt haben. Warum bist du hier, fragt sie denjenigen, von dem sie weiss, dass er einmal gesagt hat: Wir sehen uns hier im Flug der Gespräche ziemlich genau. Ich war durstig, sagt er, und habe deshalb vom Wasser getrunken, das nicht abgekocht war, und bin an Typhus gestorben. Und du, fragt sie denjenigen, von dem sie weiss, dass er jemand anderen einmal einen Schundliteraten genannt hat. Ich bin erfroren. Und du? Wenn das jemand sieht. Ich bin verhungert. Irgendein Satz fliegt in den Himmel hinauf und hat nicht mehr und nicht weniger Gewicht, als der, der ihn einmal gesagt hat."(190f.)

Das `Gefälle`, gegen das sich die Hauptfigur in den verschiedenen Lebensphasen immer wieder wehrt, lässt sich nicht aufheben. ${ }^{9}$ Was heisst das für die Protagonistin - oder für die Menschen allgemein? Resignieren? Sich damit abfinden? Oder auf bessere Zeiten im Jen-

\footnotetext{
9 Z.B. II, 121 oder III, 177: "Waren gerade sie, die Kommunisten, nicht angetreten, das Gefälle einzuebnen, damit jeder frei stehen könne, ohne zu fallen, ohne zu schieben, stossen, geschoben, gestossen zu werden, frei - ohne Angst?《
} 
seits hoffen? Eine künftige friedliche Welt sehnen sich zumindest die Verhungerten und Erfrorenen im sibirischen Exil herbei:

"Vielleicht werden auch hier, mitten in dieser Wüste, einmal Blumen wachsen, Tulpen vielleicht sogar, vielleicht wird die Anwesenheit unzähliger Schmetterlinge eines Tages einmal ebenso wirklich sein, wie es jetzt die Abwesenheit jeglicher Schmetterlinge ist, bei minus 63 Grad Celsius.«(191)

Doch wird auch diese Art von Hoffnung gleich wieder entmystifiziert, in dem sie lakonisch hinzufügt: "Sie hat nun, wie die anderen Toten, alle Zeit der Welt, um auf andere Zeiten zu warten. (191)

Was erwarten wir nach dem Tod? Jenny Erpenbeck bietet eine ganze Palette von Bildern und Vorstellungen, die ihre Figuren bestimmen und formulieren. Ist es das "Nichts", der DDR-Schriftstellerin, "die an nichts Überirdisches glaubte« (216), oder ist da doch etwas, wie die alt gewordene Frau Hoffmann und ihr Sohn in ihren letzten gemeinsamen Gesprächen zu ahnen beginnen:

"In diesem Land, in das seine Mutter übersetzt, indem sie alles, was sie jemals verstanden hat, nicht mehr verstehen kann, wird sie keine Worte mehr brauchen, so viel versteht er. Einen kurzen, hellen und scharfen Moment lang versteht er, wie es wäre, wenn er mit ihr dort ankommen könnte: Das Kornfeld wäre von Grund auf da, ebenso das Rauschen der Blätter in seinem Rücken [...], und die Erinnerung, die diese Stille ausfüllt, wäre ebenso wirklich wie die Schritte aller Menschen, die in diesem Augenblick gerade über die Erde gehen [...] - kurz, scharf und hell weiss er einen Moment lang, wie es sich anfühlen würde, wenn das Hörbare und das Unhörbare, das Ferne und das Nahe, das Innere und das Äussere, das Tote und das Lebendige gleichzeitig da wären [...], und dieser Augenblick, in dem alles gleichzeitig da wäre, würde ewig dauern.« (280f.)

\section{»Denken heisst Überschreiten«}

Jenny Erpenbeck liefert keine fertigen Antworten und schon gar keine Wahrheiten. Sie gibt uns Stoff zum Nachdenken, indem wir die Handlungen und vor allem die Gedanken ihrer Figuren mitverfolgen können. Sie weiss, was die Menschen beschäftigt; sie kennt die Ideale und Hoffnungen, die sie antreiben, ebenso wie die Zweifel und Enttäuschungen, die sie durchleben. Die Szenen, die sie 
beschreibt, sind real und lebensnah. Und doch spürt man schon von den ersten Sätzen an, dass da noch mehr mitschwingt als die snackter Realität, etwas, das darüber hinaus weist. Es ist nicht nur ihr narratives Konzept des Wiederauflebenlassens, sondern vor allem auch "ihr überempfindlicher Umgang mit Sprache«, welcher diesen Effekt erzeugt. "Zwischen den Worten, die sie so achtsam und poetisch präzise setzt, entstehen Welten aus vielfach möglichen Bedeutungen. Alles, worüber sie schreibt [...], bleibt nur zum Teil es selbst und wird zur Metapher für etwas vielfach Grösseres ${ }^{10}$, schreibt Katharina Granzin in einer Rezension treffend.

"Denken heisst Überschreiten", postuliert Ernst Bloch, und es scheint so, als entspräche die Art und Weise, wie Jenny Erpenbeck schreibt, tatsächlich seiner Vorstellung des Überschreitens, wenn er weiter ausführt:

"So jedoch, dass Vorhandenes nicht unterschlagen, nicht überschlagen wird. [...] Deshalb geht wirkliches Überschreiten auch nie ins bloss Luftleere eines Vor-uns, bloss schwärmend, bloss abstrakt ausmalend. Sondern es begreift das Neue als eines, das im bewegt Vorhandenen vermittelt ist, ob es gleich, um freigelegt zu werden, aufs Äusserste den Willen zu ihm verlangt. ${ }^{11}$

Bloch schreibt dies im Kontext eines Prozesses, der zur Hoffnung hin führt.

Aller Tage Abend ist ein Beitrag auf dem Weg zur Hoffnung; es ist ein Beitrag, der sich sehr existenziell an der "Front " abspielt und mit seiner reflektierten, »begriffenen Hoffnung" »durch das vermittelte Reich der Möglichkeit « ${ }^{12}$ führt. Nur, dass dieser Weg des Denkens und Überschreitens bei Jenny Erpenbeck - im Unterschied zu Ernst Blochs marxistischem Ideal - nicht strukturiert dialektisch nach vorne orientiert, sondern von Brüchen und Wendungen durchzogen ist. Das Ideal, die neue Welt, für die sich die Protagonistin als junge Frau und Kommunistin durchaus "zu verschwenden" (152) bereit war, erweist sich in der zweiten Hälfte des 20. Jahrhunderts als nicht umsetzbare Utopie. Es ist diese Diskrepanz - die Diskrepanz zwischen Anspruch und Wirklichkeit, zwischen Verankerung in der Vergangenheit und Aufbruch in die Zukunft, zwischen menschli-

\footnotetext{
${ }^{10}$ Katharina Granzin, Das Leben Schreiben, in: Literaturblatt für Baden-Württemberg, März/April 2013; http://www.literaturblatt.de/heftarchiv/heftarchiv-2013/22013inhaltsverzeichnis-der-gedruckten-ausgabe/das-leben-schreiben-die-autorin-jennyerpenbeck-erhaelt-den-schubart-literaturpreis-der-stadt-aalen.html (18.4.2016).

${ }^{11}$ E. Bloch, Prinzip Hoffnung, 2.

12 Vgl. ebd., 5.
} 
cher Begrenztheit und der Hoffnung auf das ewig Bleibende - welche bei Jenny Erpenbeck das Denken, oder noch treffender, das Nachdenken, antreibt. In diesem Spannungsfeld vollzieht sie eine 360-Grad-Bewegung, die das weite Spektrum der vielfältigen Möglichkeiten menschlichen Lebens auslotet, und sich doch nicht nur im Kreis bewegt, sondern verschlossene Türen öffnet und Schwellen überschreitet. Sie tut das, wie gesagt, mit ihrer Sprache und mit sich immer wieder kontrastierenden Handlungs- und Gedankengängen, die den Lesenden die Augen öffnen und sie neue Zusammenhänge erschliessen lassen.

Mit den >Wäre-jedoch-Intermezzi< überschreitet sie die Grenze schlechthin. Um - so paradox es anmutet - zur Erkenntnis zu kommen, dass der Tod eine Grenze ist, die der Mensch nicht umgehen kann.Vielmehr macht der Tod in all seinen geschilderten Facetten - als individueller Tod, als Tod des Anderen und als allgemeiner Tod - die Begrenztheit menschlicher Einflussnahme auf die individuelle Erfüllung ebenso wie auch auf eine vollendete Welt deutlich. Und so mündet der Weg der Protagonistin und ihres Sohnes im letzten Buch in eine Hoffnung, die bei einer Autorin, die sich selbst als Atheistin bezeichnet, überrascht. Es ist die Erkenntnis, als Mensch nicht alles selber wissen, erinnern, beeinflussen und vollenden zu müssen, und die fein angedeutete Hoffnung, dass es etwas Anderes, Grösseres gibt, auf das man diesbezüglich vertrauen darf.

"Weisst du, sagt sie, ich habe Angst, dass alles verlorengeht - dass die Spur verlorengeht. Welche Spur, fragt der Sohn. Ich weiss nicht mehr, woher und wohin. Der Sohn schweigt. Über den weiten Himmel ziehen ein paar Wolken. Zwei Flugzeuge, die ganz weit oben geflogen sind, haben Kondensstreifen hinterlassen, die sich jetzt langsam wieder in Himmel verwandeln.« (278)

\section{Aufgehoben in Gottes Erinnerung}

Die Fragen und Überlegungen zu Leben und Tod, zu >Was bleibt? und ,Was wird sein? ?, die im Verlauf der fünf Bücher respektive fünf Leben zur Sprache kommen, rufen danach, theologisch aufgegriffen und weiter gedacht zu werden. ${ }^{13}$ Dabei scheint der eschatologische

\footnotetext{
${ }^{13}$ Jenny Erpenbeck hat 2013 dem Evangelischen Buchpreis erhalten - gerade auch deswegen, weil der Roman anregte, "über uns selbst, unser Miteinander und unser Leben mit Gott neu nachzudenken". Aus der Laudatio von Bischof Jan Janssen, ge-
} 
Ansatz von Magdalene Frettlöh geradezu auf Jenny Erpenbecks Gedankenwelt zugeschnitten zu sein. ${ }^{14}$

Nicht nur im geschilderten Verlust des Tagebuches, auch an anderen Stellen, formuliert Jenny Erpenbeck den Wunsch, die Erinnerungen an einen Menschen wenigstens mit Worten über den Tod hinaus lebendig halten zu können. Ob dies gelingt? Vielleicht - manchmal - eine Zeit lang. Aus christlicher Sicht schöpfen wir Trost darin, dass das, was wir nicht zu leisten vermögen, von Gott vollendet wird. ER oder SIE wird sich an uns erinnern, wird unsere Namen in das himmlische Buch des Lebens einschreiben und so verewigen. ${ }^{15}$ Der Eigenname, so Frettlöh, spielt eine wichtige Rolle. ${ }^{16}$ Das zeigt schon das Taufgeschehen mit der expliziten $\mathrm{Na}$ mensnennung. Die irdische Zusage der Gotteskindschaft wird durch die Einschreibung ins Lebensbuch in ihrer bleibenden Gültigkeit bestätigt. Interessanterweise erhält die namenlose Protagonistin im fünften Buch des Romans, wo das Loslassen-Können und Vertrauen auf eine jenseitige Vollendung wächst, endlich einen Namen. Sie heisst nun - nomen est omen - Frau Hoffmann.

Neben dem Buch des Lebens ist in der Bibel auch von anderen himmlischen Büchern, zum Beispiel Tagebüchern, die Rede. ${ }^{17}$ Sie alle dienen der göttlichen Erinnerung an das irdische Sein. Bei Gott geht nichts verloren. Im Jüngsten Gericht werden die Bücher geöffnet, so

»dass alles irdische Leben so offenbar wird, wie es dem Blick Gottes sich zeigt, wie es in den Augen Gottes wahr ist. Es geht um die Erhellung menschlicher Lebensgeschichten [...]. Was undurchsichtig war, wird transparent; was im Dunkeln lag, kommt an den Tag, weil nichts mehr verborgen gehalten werden muss. $[\ldots]$

Ich hoffe darauf, dass dabei auch die nicht realisierten Möglichkeiten in den Blick kommen, dass wir erkennen, was hätte

halten am 15.05.2013; http://www.evangelischerbuchpreis.de/fileadmin/redakteur/ Buchpreis/Presse/Rede_130515_Buchpreis-final.pdf (18.4.2016).

${ }^{14}$ Magdalene L. Frettlöh, "Ja den Namen, den wir geben, schreib' ins Lebensbuch zum Leben", in: Ruth Hess und Martin Leiner, Alles in allem. Eschatologische Anstösse, Neukirchen-Vluyn 2005, 133-165.

${ }^{15}$ Vgl. ebd., 139f. "Buch des Lebens" (Phil 4,3; Apk 3,5; 13,8; 17,8; 20,12.15; 21,27).

16 Ebd., 136f.

17 Ebd., 152f.;Vgl. ebd., 140: "Buch der Lebendigen« (Ps 69,29); Gedächtnisbuch Gottes (Mal 31,16); Buch Gottes mit den Namen der Lebenden (Ex 32,32f.; Dan 12,1); Buch mit den Lebenstagen (Ps 139,16); Buch, in dem die Tränen verzeichnet sind (Ps 56,9); himmlisches Namensverzeichnis (Lk 10,20; Hebr 12,23). 
sein können, wenn wir uns etwa in dem einen oder anderen Fall anders entschieden hätten, anderen Menschen begegnet, an einem anderen Ort gewesen wären ... [...] Wenn Gott das, was nicht ist, ins Leben ruft (Röm 4,17), wird SIE dann nicht auch das Unabgegoltene der Vergangenheit neu inszenieren, das, was hätte sein können, zu dem machen, "was hat sein werden ${ }^{18}$. Damit würde derVergangenheit (und nicht nur der Gegenwart!) eine offene Zukunft geschenkt [...]. (19 $^{19}$

Diese Hoffnungsbotschaft könnte direkt an die Figuren in Jenny Erpenbecks Roman gerichtet sein. Es ist eine Botschaft, die den Verlust und die Trauer über all die ungelebten Möglichkeiten, über "alles, was aus dem Kind hätte werden können«(11), ernst nimmt und ihnen einen Sinn zuspricht. Es ist eine Botschaft, die das Loslassen leichter macht. In diese Richtung könnten auch die Gespräche von Frau Hoffmann und ihrem Sohn am Ende des Romans gedeutet werden. Am Schluss akzeptiert Frau Hoffmann, dass ihre Möglichkeiten in dieser Welt - auch wenn es immer noch etwas zu realisieren gäbe - ausgeschöpft sind, und sie stirbt nun endgültig. Sie stirbt, weil sie hoffen kann, dass dies noch nicht alles war.

Zwischen all den Fragen und unbekannten Antworten, zwischen dem Nicht-Wissen und der Gewissheit gibt es ein >Mittleres`: die Hoffnung. Zu dieser hat uns Jenny Erpenbeck mit Aller Tage Abend hingefuihrt und damit tatsächlich so etwas wie eine skleine Eschatologie geschrieben.

- Monika Hirt hat Germanistik, Philosophie und Ethnologie studiert. Zurzeit ist sie Theologiestudentin an der Universität Zürich und schreibt gerade ihre Bachelorarbeit über das hier vorgestellte Thema.

${ }^{18}$ Frettlöh zitiert hier: Jürgen Ebach, Eine fehlende Zeitform in der deutschen Grammatik. Kleiner ungehaltener Zwischenruf, in: Ders., Ein weites Feld - ein zu weites Feld?, Bochum 2004, 218.

19 Ebd., 159 f. 